\title{
Impacto Positivo do Exercício no Índice Tornozelo Braquial em Pacientes Portadores de Doença Renal Crônica
}

Giardin, G.; Duarte, G. A.; Vargas, M. M.; Gastaud, L.; Schuch, A.; Bohlke, M.

Hospital Universitário São Francisco de Paula, Pelotas, RS, Brasil

\section{Resumo}

INTRODUÇÃO: As repercussões da insuficiência renal crônica são observadas em todos os sistemas do corpo, levando a uma pior qualidade de vida. Treinamentos aeróbicos são propostas seguras e eficazes para portadores de doença renal crônica. Estudos demonstraram melhora na condição física e diminuição da pressão arterial sistólica de repouso após um programa de treinamento por exercícios. A incidência de eventos cardiovasculares tem apresentado um crescimento exponencial com o passar dos anos. Cerca de noventa por cento dos pacientes com doença arterial coronariana (DAC) tem pelo menos um fator de risco de aterosclerose. Muitos estudos comprovam o potencial da aferição do índice tornozelo braquial (ITB) como marcador de aterosclerose e de doença arterial oclusiva periférica, e várias pesquisas demonstram sua utilidade na estratificação de risco cardiovascular. MÉTODOS: Ensaio clínico randomizado e controlado que avaliou o efeito do exercício em uma amostra de 150 pacientes não diabéticos portadores de hipertensão arterial, foi conduzida uma análise de subgrupo em 64 (42.95\% da amostra total) indivíduos portadores de doença renal crônica, definida como uma taxa de filtração glomerular menor ou igual a $60 \mathrm{~mL} / \mathrm{min} / 1,74 \mathrm{~m} 2$. O subgrupo foi composto por 28 (43.08\%) indivíduos de sexo masculino e 37 (56.92\%) do sexo feminino, com idade de 68.81 (10.51) anos. A intervenção foi constituída por um programa estruturado de 
exercícios aeróbicos e de resistência aplicado durante um período de 16 semanas. Foi avaliado o efeito do exercício em um conjunto de marcadores substitutos de doença cardiovascular, avaliados antes e após o período de intervenção, constituído por glicemia de jejum, perfil lipídico, proteína $\mathrm{C}$ reativa ultrassensível, filtração glomerular estimada a partir de creatinina plasmática, índice de massa corporal e índice tornozelo braquial (ITB). RESULTADOS: Foi detectado efeito significativo do exercício físico no ITB, com uma variação de -,072 (IC 95\% -,183 a ,039) no grupo controle e um aumento de ,09 (IC95\% -,003 a ,184) no grupo intervenção ( $\mathrm{p}=0,01)$. Não foi detectado efeito do exercício nos demais marcadores de DCV nesse subgrupo de pacientes portadores de DRC. CONCLUSÃO: O ITB tem sido considerado um marcador de DCV e sua redução associada a aumento da mortalidade na população geral. Essa análise de subgrupo sugere um potencial impacto positivo do exercício físico estruturado no ITB em pacientes portadores de DRC, grupo de elevado risco cardiovascular. 